\title{
Frames of subspaces in Hilbert spaces with $W$-metrics
}

\author{
Primitivo Acosta-Humánez, Kevin Esmeral, Osmin Ferrer
}

\begin{abstract}
In this paper we start considering a sesquilinear form $\langle W \cdot, \cdot\rangle$ defined over a Hilbert space $(\mathfrak{H},\langle\cdot, \cdot\rangle)$ where $W$ is bounded $\left(W^{*}=W \in \mathcal{B}(\mathfrak{H})\right)$ and ker $W=\{0\}$. We study the dynamic of frame of subspaces over the completion of $(\mathfrak{H},\langle W \cdot, \cdot\rangle)$ which is denoted by $\mathfrak{H}_{W}$ and is called Hilbert space with $W$-metric or simply $W$-space. The sense of dynamics studied here refers to the behavior of frame of subspaces comparing $\mathfrak{H}_{W}$ with $\mathfrak{H}$ as well $\mathfrak{H}$ with $\mathfrak{H}_{W}$. Furthermore, we show that for any Hilbert space with $W$-metric $\mathfrak{H}_{W}$, being 0 an element of the spectrum of $W(0 \in \sigma(W))$, has a decomposition $\mathfrak{H}_{W}=\bigoplus_{n \in \mathbb{N} \cup\{\infty\}} \mathfrak{H}_{\psi_{n}}^{W}$, where $\mathfrak{H}_{\psi_{n}}^{W} \simeq L_{2}\left(\sigma(W), x d \mu_{n}(x)\right)$ for all $n \in \mathbb{N} \cup\{\infty\}, L_{2}$ denotes a Hilbert space square integrable and $\mu$ a Lebesgue measure. Finally, the case when $W$ is unbounded also considered.
\end{abstract}

\section{Introduction}

The theory of frames in Hilbert spaces provides a flexible alternative due to allows to avoid linear independence and ortogonality bettween its elements. This theory was introduced by Duffin and Schaeffer in 1952, see [14], leading to new developments and applications in functional analysis and related areas, see for example $[8,9,10,11,13,15,16,17]$. Moreover, in the papers of Cazzasa, Kutniok and Găvruţa have been studied the frame of subspaces or

Key Words: frame of subspaces; Dual frame, Fusion frames; frames in Krein spaces; Krein spaces; frames in $W$-spaces.

2010 Mathematics Subject Classification: Primary 42C15, 46C20, 47B50, 47B15.

Received: January, 2014.

Revised: March, 2014.

Accepted: March, 2014. 
Dual frames, see [9, 17], while Hilbert spaces with $W$-metric were studied by Azizov and Iokhvidov, see [5]. Recently in [15, 16], works involving second and third author, have been proven that the properties $0 \in \rho(W)$ (i.e., 0 is in the resolvent of $W), 0 \in \sigma(W)$ (i.e., 0 is in the spectrum of $W$ ) have influence over the behavior of the frames of subspaces.

Inspired by the theory of $C^{*}$-algebra, where the theorem of spectral representation (see [20]) is proved for Hilbert spaces, in this paper we define frame of subspaces on Hilbert spaces with $W$-metric, analyzing the influence of the properties of $W$, concerning to its spectrum and its resolvent, over such frame of subspaces. Furthermore, as aim of this paper, we rewrite this important theorem in the sense of frame of subspaces as well in the context of Hilbert spaces with a $W$-metric.

The starting point of this paper is the theoretical background related to Krein spaces and Hilbert spaces with $W$-metric. Afterwards, we present our approach to the study of frame of subspaces. In particular, we introduce the concepts of frame of subspaces in Hilbert spaces and frame of subspaces in Krein spaces. The last one coming from the definition of frame in Krein spaces given in [15], being some results derived from such definition. Next, is considered thereof way the frames of subspaces in Hilbert spaces with $W$ metrics when the Gram operator $W$ is bounded, although $0 \in \sigma(W)$ as well for $0 \notin \sigma(W)$. Later, are analyzed the frames of subspaces over regular and singular Krein space (respectively). The main result of this paper is such as follows: any singular Krein space has a decomposition in direct sum of singular Krein subspaces $\mathfrak{H}_{\psi_{n}}^{W}$, which are isomorphic to $L_{2}\left(\sigma(W), x d \mu_{n}(x)\right)$ for every $n \in \mathbb{N} \cup\{\infty\}$. Finally we study the behavior of the frame of subspaces when the Gram operator is unbounded. The interested reader on these subjects can found some open questions and remarks as well complementary references ending the paper.

\section{Preliminaries}

Definition 1.1 (Krein Spaces). Let $\Re$ and $[\cdot, \cdot]: \Re \times \Re \longrightarrow \mathbb{C}$ be a $\mathbb{C}$-vector space and a sesquilinear form respectively. The vector space $(\Re,[\cdot, \cdot])$ is a Krein space whether $\Re=\Re^{+} \oplus \Re^{-}$and $\left(\Re^{+},[\cdot, \cdot]\right)$, $\left(\Re^{-},-[\cdot, \cdot]\right)$ are Hilbert spaces, being $\Re^{+}$and $\Re^{-}$orthogonals with respect to $[\cdot, \cdot]$.

We define the following scalar product over $\Re$ :

$$
\left(x_{1}, x_{2}\right)=\left[x_{1}^{+}, x_{2}^{+}\right]-\left[x_{1}^{-}, x_{2}^{-}\right], x_{i}^{ \pm} \in \Re^{ \pm}, x_{i}=x_{i}^{+}+x_{i}^{-} .
$$

We can see that $(\Re,(\cdot, \cdot))$ is a Hilbert space, the so-called Hilbert space associated to $\Re$. Henceforth, the orthogonal projections over $\Re^{+}$and $\Re^{-}$will be 
denoted by $P^{+}$and $P^{-}$respectively. Likewise, the linear bounded operator $J=P^{+}-P^{-}$, the so-called Fundamental Symmetry, satisfies the property: $[x, y]=(J x, y), \forall x, y \in \Re$. We can see that this property can be written as

$$
[x, y]_{J}=[J x, y]=(x, y), \quad\|x\|_{J}:=\sqrt{[x, x]_{J}} \forall x, y \in \Re .
$$

Definition 1.2. Let $(\Re,[\cdot, \cdot])$ be a Krein space and consider $x, y \in \Re$. We say that $x$ is orthogonal to $y$, denoted by $x \perp y$, whether $[x, y]_{J}=0$. Similarly, we say that $x$ is $J$-orthogonal to $y$, denoted by $x[\perp] y$, whether $[x, y]=0$.

Definition 1.3. Consider a Krein space $\Re$ and a closed subspace $V$ of $\Re$. The subspace

$$
V^{[\perp]}=\{x \in \Re:[x, y]=0, \text { for all } y \in V\}
$$

is the so-called $J$-orthogonal complement of $V$ with respect $[\cdot, \cdot]$ (or simply $J$-orthogonal complement of $V$ ).

Definition 1.4. A closed subspace $V$ of $\Re$ satisfying $V \cap V^{[\perp]}=\{0\}$ and $V+V^{[\perp]}=\Re$, being $V^{[\perp]}$ as in $(1.2)$, is called closed subspace projectively complete.

Remark 1.5. From now on, any closed subspace $V$ considered in this paper will be projectively complete. We denote by $P_{V}$ and $Q_{V}$ the orthogonal and $J$-orthogonal projections on $V$ respectively. i.e., $P_{V}^{* J}=P_{V}=P_{V}^{2}$ and $Q_{V}^{[*]}=$ $Q_{V}=Q_{V}^{2}$. On the other hand, in [6] was proven that if $V$ is a closed subspace, then their $J$-orthogonal complement $V^{[\perp]}$ and orthogonal complement $V^{\perp}$ are closed subspaces. Therefore, such subspaces are linked by the formulas

$$
V^{[\perp]}=J V^{\perp}, \quad V^{\perp}=J V^{[\perp]}, \quad(J V)^{[\perp]}=J V^{[\perp]} .
$$

$B y$ (1.3) we note that $J V$ is projectively complete if and only if $V$ is projectively complete. Moreover, condition $V \cap V^{[\perp]}=\{0\}$ set that for any $k \in \Re, k$ has an unique $J$-orthogonal projection over $V$, see [6] for complete statements and proofs.

Remark 1.6. Let $(\Re,[\cdot, \cdot])$ and $V$ be a Krein space and a projectively complete closed subspace of $\Re$ respectively. In consequence, $V=\bar{V}=\left(V^{[\perp]}\right)^{[\perp]}$ which implies that $(V,[\cdot, \cdot])$ is a Krein space. Hence $V=V^{+}+V^{-}$, where $V^{+} \subset \Re^{+}$, and $V^{-} \subset \Re^{-}$and we conclude that $J V \subset V$.

Definition $1.7([6])$. Let $\Re$ and $\left\{e_{i}\right\}_{i \in I} \subset \Re$ be a Krein space and a system of vectors respectively, where $I$ is an arbitrary set of indices. If $\left[e_{i}, e_{j}\right]= \pm \delta_{i, j}$ for all $i, j \in I$, where $\delta_{i, j}$ is the Kronecker's delta, then such system of vectors is named $J$-orthonormalized system. 
Example 1.8. Assume that $E_{+}=\left\{e_{i}^{+}\right\}_{i \in I}$ and $E_{-}=\left\{e_{k}^{-}\right\}_{k \in K}$ are $J$ orthonormalized system of $\Re^{+}$and $\Re^{-}$respectively. The system of vectors $E=E_{+} \cup E_{-}$is a J-orthonormalized system of $\Re$.

Definition $1.9([6])$. A J-orthonormalized system is called maximal whether if it is not contained in any wider $J$-orthonormalized system, and to be $J$ complete whether there is no non-zero vector J-orthonormalized to this system.

Definition $1.10([6])$. Let $(\Re,[\cdot, \cdot])$ be a Krein space. A J-orthonormalized basis in $\Re$ is a $J$-orthonormalized system, $J$-complete and maximal in $\Re$.

Remark 1.11. Since $\left(\Re,[\cdot, \cdot]_{J}\right)$ is a Hilbert space, we can study linear operators acting on Krein spaces. Some topological concepts such as continuity and closure over operators and spectral theory, are concerning to the topology induced by the $J$-norm given in (1.1). Therefore, some definitions related with operator theory in Hilbert spaces are satisfied in a more general way. For example, we can consider the space of all bounded operators on $\Re$

$$
\mathcal{B}(\Re)=\left\{T: \Re \rightarrow \Re: \text { lineal y }\|T\|=\sup _{x \in \Re \backslash\{0\}} \frac{\|T x\|_{J}}{\|x\|_{J}}<\infty\right\} .
$$

The adjoint of an operator $T$ in Krein spaces, denoted by $T^{[*]}$, satisfies $[T(x), y]=\left[x, T^{[*]}(y)\right]$. However, such $T$ have an adjoint operator in the Hilbert space $\left(\Re,[\cdot, \cdot]_{J}\right)$, denoted by $T^{* J}$, where $J$ is the fundamental symmetry in $\Re$. Furthermore, there is a relation between $T^{* J}$ and $T^{[*]}$, which is $T^{[*]}=J T^{* J} J$. Moreover, let $\Re$ and $\Re^{\prime}$ be Krein spaces with fundamental symmetries $J_{\Re}$ and $J_{\Re^{\prime}}$ respectively, if $T \in \mathcal{B}\left(\Re, \Re^{\prime}\right)$, then $T^{[*]_{\Re}}=J_{\Re} T^{* J_{\Re}} J_{\Re^{\prime}}$.

The following definition is in agreement with the previous remark.

Definition 1.12. An operator $T \in \mathcal{B}(\Re)$ is called self-adjoint whether $T=$ $T^{[*]}$ and is called Jself-adjoint whether $T=T^{* J}$. Moreover, a linear operator $T$ is called positive whether $[T k, k] \geq 0$ for all $k \in \Re$. An operator $T$ is called uniformly positive whether there exists $\alpha>0$ such that $[T k, k] \geq \alpha\|k\|_{J}$ for all $k \in \Re$.

The next result will be used along this paper and its proof is similar to the case of Hilbert spaces, see [17].

Proposition 1.13. Let $\Re$ and $\widetilde{\Re}$ be Krein spaces with fundamentals symmetries $J, \widetilde{J}$ respectively. Consider $V \subset \Re$ a closed subspace with $J$ orthogonal projection $Q_{V}: \Re \rightarrow V$, and orthogonal projection $P_{V}: \Re \rightarrow V$. Let $\mathcal{U}:\left(\Re,[\cdot, \cdot]_{J}\right) \rightarrow\left(\widetilde{\Re},[\cdot, \cdot]_{\widetilde{J}}\right)$ and $\mathcal{T}: \Re \rightarrow \widetilde{\Re}$ be unitary operators. Then

$$
\mathcal{U} P_{V} \mathcal{U}^{-1}=P_{U V}, \quad \mathcal{T} Q_{V} \mathcal{T}^{-1}=Q_{\mathcal{T} V},
$$


where $P_{\mathcal{U} V}: \widetilde{\Re} \rightarrow \mathcal{U} V$ is the orthogonal projection on $\mathcal{U} V$, and $Q_{\mathcal{T} V}: \widetilde{\Re} \rightarrow \mathcal{T} V$ is the $J$-orthogonal projection on $\mathcal{T} V$. In particular, if $\Re=\widetilde{\Re}$ and $J=\widetilde{J}$, then

$$
P_{J V}=J P_{V} J=P_{V}^{[*]}, \quad Q_{J V}=J Q_{V} J=Q_{V}^{* J} .
$$

Proposition 1.14. Let $V$ be a closed subspace of . The following statements hold.

i). If $P_{V}$ is an orthogonal projection on $V$, then $Q_{V}=P_{J V} P_{V}$ is a $J$ orthogonal projection on $V$.

ii). If $Q_{V}$ is an $J$-orthogonal projection on $V$, then $P_{V}=Q_{J V} Q_{V}$ is an orthogonal projection on $V$.

Proof. We prove only item $i$ ) due to the proof of item $i$ ) can be done in an analogous way. Let $P_{V}$ be an orthogonal projection on $V$, consider $Q_{V}=$ $P_{J V} P_{V}$ which is defined by (1.3). Now, by (1.5) we have that $Q_{V}^{[*]}=Q_{V}$ and for instance $\left[Q_{V} x, y\right]=\left[P_{J V} P_{V} x, y\right]=\left[P_{V} x, P_{V} y\right]=[x, y]$, for all $x, y \in V$. Thus, $Q_{V} x=x, \forall x \in V$. On the other hand, since $J V \subset V$, we get $Q_{V}^{2}=$ $P_{J V} P_{V} P_{J V} P_{V}=P_{J V}^{2} P_{V}=P_{J V} P_{V}=Q_{V}$.

\subsection{Hilbert spaces with $W$-metrics}

Definition 1.15 ( $W$-metrics).

Let $\mathfrak{H}$ be a Hilbert space with scalar product $\langle\cdot, \cdot\rangle$, and induced norm $\|\cdot\|=$ $\sqrt{\langle\cdot, \cdot\rangle}$. Consider the operator $W=W^{*} \in \mathcal{B}(\mathfrak{H})$ with $\operatorname{ker} W=\{0\}$. The sesquilinear form

$$
[\cdot, \cdot]=\langle W(\cdot), \cdot\rangle
$$

defined on $\mathfrak{H}$ is so called $W$-metric, or, $W$-inner product, this operator $(W)$ is called the Gram operator.

Proposition 1.16. A Hilbert space with a $W$-metric can be densely embedded in a Krein space $\mathfrak{H}_{W}$ with fundamental symmetry $J$.

Proof. See [5].

Remark 1.17 (Consequences given by the Gram operator). Let $W$ be the Gram operator over $\mathfrak{H}$.

i). If $0 \in \rho(W)$, then

$$
\left\|W^{-1}\right\|^{-1}\|x\|^{2} \leq\|x\|_{J}^{2} \leq\|W\|\|x\|^{2}, \quad \forall x \in \mathfrak{H} .
$$

Therefore

$$
\mathfrak{H}_{W}=\overline{\left(\mathfrak{H},[\cdot, \cdot]_{J}\right)} \|^{\|\cdot\|_{J}}=\left(\mathfrak{H},[\cdot, \cdot]_{J}\right) .
$$


ii). If $0 \in \sigma(W)$, then

$$
\|x\|_{J} \leq \sqrt{\|W\|}\|x\|, \quad \forall x \in \mathfrak{H} .
$$

Hence

$$
\mathfrak{H}_{W}:=\overline{\mathfrak{H}}^{\|\cdot\|_{J}} .
$$

Definition 1.18. Let $(\mathfrak{H},\langle\cdot, \cdot\rangle)$ be a Hilbert space. The Krein space $\mathfrak{H}_{W}$ is called regular whether the Gram operator $W$ is such that $0 \in \rho(W)$. Otherwise is called singular.

More details of the regular and singular Krein spaces can be found in [5].

Remark 1.19. Consider the polar decomposition of $W$ given by the formula

$$
W=J|W|,
$$

where the linear operator $J:(\operatorname{ker}|W|)^{\perp}=\overline{\text { Rang }|W|}=\mathfrak{H} \rightarrow \overline{\operatorname{Rang} W}=\mathfrak{H}$ is a partial isometry. However, ker $J=\{0\}$, this imply that $J$ is a unitary operator.

Proposition 1.20. The operators $|W|, W$ commute with $J$, where $J$ is such that (1.11) is true. Also $J=J^{*}$.

Proof. By the properties of the spectral measure we have $W|W|=|W| W$. Hence, $(J|W|-|W| J)|W|=0$. i.e $J|W|=|W| J$. On the other hand, note that $J|W|=W=W^{*}=|W| J^{*}$. Therefore $|W|\left(J-J^{*}\right)=0$. i.e., $J=J^{*}=$ $J^{-1}$, because $\operatorname{ker}|W|=\{0\}$. Since $J|W|=|W| J$, we have $J W=J(J|W|)=$ $J(|W| J)=J|W| J=W J$.

Definition 1.21. The space $\mathfrak{H}_{W}$ is a Krein space with the J-norm generated by the inner product

$$
[x, y]_{J}=[J x, y]=\langle W J x, y\rangle=\langle|W| x, y\rangle \quad \forall x, y \in \mathfrak{H},
$$

where $J$ is the symmetry of Hilbert space $\mathfrak{H}$ such that $W=J|W|$.

\section{Main results}

In this section we present an approach to study the frames of subspaces in the context of Krein and Hilbert spaces. 


\subsection{Frame of subspaces on Hilbert spaces}

Next, we consider the frames of subspaces in Hilbert spaces. See details in [9]. From now on we consider $I$ like a set of indices, and define

$$
\ell_{+}^{\infty}(I)=\left\{\left(x_{i}\right)_{i \in I} \in \ell^{\infty}(I): x_{i} \in \mathbb{R}_{+}, \quad \forall i \in I\right\} .
$$

Definition 2.1 (Frame of subspaces). Let $\mathfrak{H}$ be a Hilbert space with norm $\|\cdot\|$. A family $\left\{V_{i}\right\}_{i \in I}$ of closed subspaces of $\mathfrak{H}$ is called a frame of subspaces of the Hilbert space $\mathfrak{H}$ with respect $\left(x_{i}\right)_{i \in I} \in \ell_{+}^{\infty}(I)$ (denoted by $\left\{x_{i}, V_{i}\right\}_{i \in I}$ ) whether there are constants $A, B>0$ such that

$$
A\|y\|^{2} \leq \sum_{i \in I} x_{i}^{2}\left\|P_{V_{i}} y\right\|^{2} \leq B\|y\|^{2}, \forall y \in \mathfrak{H}
$$

being $P_{V_{i}}: \mathfrak{H} \rightarrow V_{i}$ orthogonal projections. The numbers $A$ and $B$ are called frame bounds.

\subsection{Frame of subspaces on Krein spaces}

Now, we consider the frame of subspaces in Krein spaces. This work is based in the properties given in the case Hilbert spaces, which can be found in [9] and see also [15].

Definition 2.2. Let $\Re$ be a Krein space with fundamental symmetry J. Consider a family of closed subspaces $\left\{V_{i}\right\}_{i \in I}$ of $\Re$ with $Q_{V_{i}}: \Re \rightarrow V_{i}$ their respective $J$-orthogonal projections. Fix $\left(x_{i}\right)_{i \in I} \in \ell_{+}^{\infty}(I)$, we say that $\left\{x_{i}, V_{i}\right\}_{i \in I}$ is $a$ frame of subspaces of the Krein space whether there are constants $A, B>0$ such that

$$
A\|k\|_{J}^{2} \leq \sum_{i \in I} x_{i}^{2}\left\|Q_{V_{i}} k\right\|_{J}^{2} \leq B\|k\|_{J}^{2}, \forall k \in \Re .
$$

Remark 2.3. According to the previous definition and in the same way as the case of Hilbert spaces, the constants $A$ and $B$ are called frame bounds. Whenever $A=B$, the family $\left\{x_{i}, V_{i}\right\}_{i \in I}$ is called a B-tight frame of subspaces. In particular, if $A=B=1$, then the family $\left\{x_{i}, V_{i}\right\}_{i \in I}$ is called Parseval frame of subspaces. The family $\left\{x_{i}, V_{i}\right\}_{i \in I}$ is called orthonormal basis of subspaces when

$$
\Re=\bigoplus_{i \in I} V_{i}
$$

Moreover, a frame of subspaces $\left\{x_{i}, V_{i}\right\}_{i \in I}$ is called $x$-uniform, whenever $x:=$ $x_{i}=x_{j}$ for all $i, j \in I$. In the case that we only have the upper bound, the family $\left\{x_{i}, V_{i}\right\}_{i \in I}$ is called Bessel sequence of subspaces with Bessel bound $B$. 
Theorem 2.4 (Equivalence of the frames of subspaces). Let $\Re$ be a Krein space with fundamental symmetry $J$. Consider a family of closed subspaces $\left\{V_{i}\right\}_{i \in I}$ of $\Re$ with $Q_{V_{i}}: \Re \rightarrow V_{i}$ and $P_{V_{i}}: \Re \rightarrow V_{i}$ their $J$-orthogonal and orthogonal projections respectively. Fixed $\left(x_{i}\right)_{i \in I} \in \ell_{+}^{\infty}(I)$, the following statements are equivalent:

i). $\left\{x_{i}, V_{i}\right\}_{i \in I}$ is a frame of subspaces of $\Re$ with frame bounds $A, B$;

ii). $\left\{x_{i}, J V_{i}\right\}_{i \in I}$ is a frame of subspaces of $\Re$ with frame bounds $A, B$;

iii). $\left\{x_{i}, V_{i}\right\}_{i \in I}$ is a frame of subspaces of $\left(\Re,[\cdot, \cdot]_{J}\right)$ with frame bounds $A, B$;

$i v) .\left\{x_{i}, J V_{i}\right\}_{i \in I}$ is a frame of subspaces of $\left(\Re,[\cdot, \cdot]_{J}\right)$ with frame bounds $A, B$.

Proof. The equivalence of $i$ and $i i$ follows from

$$
\left\|Q_{J V_{i}} k\right\|_{J}^{2}=\left\|J Q_{J V_{i}} k\right\|_{J}^{2}=\left\|Q_{V_{i}} J k\right\|_{J}^{2} .
$$

The same argument is applied to $\left\{x_{i}, J V_{i}\right\}_{i \in I}$, together with the proposition 1.13 , to prove the equivalence of $i i i$ and $i v$, i.e.,

$$
\left\|P_{J V_{i}} k\right\|_{J}^{2}=\left\|J P_{J V_{i}} k\right\|_{J}^{2}=\left\|P_{V_{i}} J k\right\|_{J}^{2} .
$$

The equivalence $i$ and $i v$ is proved with the proposition 1.14 as follows: Given $P_{J V_{i}}$ and $P_{V_{i}}$ orthogonal projections on $J V_{i}$ and $V_{i}$ respectively, we define $Q_{V_{i}}=P_{J V_{i}} P_{V_{i}}$, which is a $J$-orthogonal projection on $V_{i}$. Thus, since $J_{V_{i}} \subset V_{i}$ we get

$$
\left\|Q_{V_{i}} k\right\|_{J}^{2}=\left\|J P_{V_{i}} J P_{V_{i}} k\right\|_{J}^{2}=\left\|P_{V_{i}} P_{J V_{i}} J k\right\|_{J}^{2}=\left\|P_{J V_{i}} J k\right\|_{J}^{2}
$$

Proposition 2.5. Fix $\left\{x_{i}\right\}_{i \in I} \in \ell_{+}^{\infty}(I)$. Consider a partition $\left\{J_{i}\right\}_{i \in I}$ of $I$ such that $I=\bigsqcup_{i \in I} J_{i}$ and $\left\{k_{i, j}\right\}_{j \in J_{i}}$ is a sequence of frame for the Krein space $\Re$ with frame bounds $A_{i}, B_{i}>0$. Define $V_{i}=\operatorname{span}_{j \in J_{i}}\left\{k_{i, j}\right\}$ for all $i \in I$ and choose an $J$-orthonormal basis $\left\{e_{i, j}\right\}_{j \in J_{i}}$ for each subspace $V_{i}$. Suppose that $0<A=\inf _{i \in I} A_{i} \leq B=\sup _{i \in I} B_{i}$, then the following statements are equivalent.

i). $\left\{x_{i} k_{i, j}\right\}_{i \in I, j \in J_{i}}$ is a frame for the Krein space $\Re$.

ii). $\left\{x_{i} e_{i, j}\right\}_{i \in I, j \in J_{i}}$ is a frame for the Krein space $\Re$.

iii). $\left\{x_{i}, V_{i}\right\}_{i \in I}$ is a frame of subspaces for the Krein space $\Re$. 
Proof. Since $\left\{k_{i, j}\right\}_{j \in J_{i}}$ is a sequence of frame for the Krein space $\Re$ with frame bounds $A_{i}, B_{i}>0$, then in [15] shown that is equivalent to say that $\left\{k_{i, j}\right\}_{j \in J_{i}}$ is a sequence of frame for the Hilbert space $\left(\Re,\|\cdot\|_{J}\right)$ with same frame bounds. Thus, by the theorem 2.4 the proof is analogous as in the case of Hilbert space, see $[9]$.

Proposition 2.6. Let $\Re$ and $\widetilde{\Re}$ be Krein spaces with fundamental symmetries $J$ and $\widetilde{J}$ respectively. Consider $\mathcal{U}:\left(\Re,[\cdot, \cdot]_{J}\right) \rightarrow\left(\widetilde{\Re},[\cdot, \cdot]_{\widetilde{J}}\right)$ an invertible operator. The family $\left\{x_{i}, V_{i}\right\}_{i \in I}$ is a frame of subspaces for the Hilbert space $\left(\Re,[\cdot, \cdot]_{J}\right)$ if and only if $\left\{x_{i}, \mathcal{U} V_{i}\right\}_{i \in I}$ is a frame of subspaces for the Hilbert $\operatorname{space}\left(\widetilde{\Re},[\cdot, \cdot]_{\widetilde{J}}\right)$.

Proof. By the proposition 1.13 we have that

$$
\left\|P_{V_{i}} k\right\|_{J}^{2}=\left\|\mathcal{U}^{-1} P_{\varkappa V_{i}} \mathcal{U}\right\|_{J}^{2}=\left\|P_{U_{V_{i}}} \mathcal{U}\right\|_{\widetilde{J}}^{2} .
$$

\subsection{Frame of subspaces in Hilbert spaces with $W$-metric}

In $[15]$ is proved that the behavior of the frames in Hilbert spaces with a $W$ metric is depending of the properties $0 \in \rho(W)$ or $0 \in \sigma(W)$. Next, we study the frames of subspaces in this spaces.

\subsubsection{Frame of subspaces in regular Krein spaces}

Theorem 2.7. Let $\mathfrak{H}_{W}$ be a regular Krein space, and $\left\{V_{i}\right\}_{i \in I}$ a family of closed subspaces of $\mathfrak{H}$. The family $\left\{x_{i}, V_{i}\right\}_{i \in I}$ is a frame of subspaces for the Hilbert space $(\mathfrak{H},\langle\cdot, \cdot\rangle)$ if and only if $\left\{x_{i}, V_{i}\right\}_{i \in I}$ is a frame of subspaces for the regular Krein space $\mathfrak{H}_{W}$.

Proof. Setting $Q_{V_{i}}=P_{J V_{i}} P_{V_{i}}$, by (1.7)

$$
\begin{aligned}
\left\|W^{-1}\right\|^{-1} \sum_{i \in I} x_{i}^{2}\left\|P_{J V_{i}} J k\right\|^{2} & \leq \sum_{i \in I} x_{i}^{2}\left\|P_{V_{i}} P_{J V_{i}} J k\right\|_{J}^{2}=\sum_{i \in I} x_{i}^{2}\left\|Q_{V_{i}} k\right\|_{J}^{2} \\
& \leq\|W\| \sum_{i \in I} x_{i}^{2}\left\|P_{J V_{i}} J k\right\|^{2} .
\end{aligned}
$$

$\Rightarrow]$ Suppose that $\left\{x_{i}, V_{i}\right\}_{i \in I}$ is a frame of subspaces for the Hilbert space $(\mathfrak{H},\langle\cdot, \cdot\rangle)$ with frame bounds $A, B$, then, by inequality $(2.5)$ obtain $\left\{x_{i}, J V_{i}\right\}_{i \in I}$ is a frame of subspaces for the Krein space $\mathfrak{H}_{W}$ with frame bounds $A^{\prime}=$ $\left\|W^{-1}\right\|^{-1} A$ and $B^{\prime}=\|W\| B$. By the theorem 2.4 we say that $\left\{x_{i}, V_{i}\right\}_{i \in I}$ is a frame of subspaces for the regular Krein space $\mathfrak{H}_{W}$.

$[\Leftarrow$ The proof is analogous as in the previous case. 


\subsubsection{Frame of subspaces in singular Krein spaces}

Theorem 2.8. Let $\mathfrak{H}_{W}$ be a singular Krein space, and $\left\{V_{i}\right\}_{i \in I}$ a family of closed subspaces of $\mathfrak{H}$. If $\left\{x_{i}, V_{i}\right\}_{i \in I}$ is a frame of subspaces for the Hilbert space $(\mathfrak{H},\langle\cdot, \cdot\rangle)$, then $\left\{x_{i}, V_{i}\right\}_{i \in I}$ is not frame of subspaces for the singular Krein space $\mathfrak{H}_{W} \cdot$

Proof. Since $\mathfrak{H}_{W}$ is a singular Krein space, then $0 \in \sigma_{c}(W)$. Thus, given $\varepsilon>0$, the spectral measure $\mathbf{E}_{\lambda}$, where $W=\int_{\sigma(W)} \lambda d \mathbf{E}_{\lambda}$, satisfies $\mathbf{E}_{\lambda}((0, \varepsilon]) \neq 0$. But $(0, \varepsilon]=\bigcup_{n \in \mathbb{N}}\left[\frac{\varepsilon}{n}, \varepsilon\right]$, hence, there is $n_{0} \in \mathbb{N}$ such that $\mathbf{E}_{\lambda}\left(\left[\frac{\varepsilon}{n_{0}}, \varepsilon\right]\right) \neq 0$. Now, we assume $f \in \mathbf{E}_{\lambda}\left(\left[\frac{\varepsilon}{n_{0}}, \varepsilon\right]\right) \mathfrak{H} \cap V_{j}$ for some $j \in I$ such that $\|f\|=1$ and $\|f\|_{J} \leq 1$ (since $\left.\|f\|_{J} \leq\|\sqrt{|W|}\|\|f\|\right)$. In this way, if $M=\sup _{j \in I} x_{j}$, then

$$
\begin{aligned}
\sum_{i \in I} x_{i}^{2}\left\|Q_{V_{i}} f\right\|_{J}^{2} & =x_{j}^{2}\|\sqrt{|W|} f\|^{2} \leq A^{-1} x_{j}^{2} \sum_{i \in I} x_{i}^{2}\left\|P_{V_{i}} \sqrt{|W|} f\right\|^{2} \\
& \leq A^{-1} B M^{2}\left\langle\left(\int_{\sigma(W)}|\lambda| d \mathbf{E}_{\lambda}\right) \mathbf{E}_{\lambda}\left(\left[\frac{\varepsilon}{n_{0}}, \varepsilon\right]\right) f, f\right\rangle \\
& =A^{-1} B M^{2} \int_{\sigma(W)}|\lambda| \chi_{\left[\frac{\varepsilon}{n_{0}}, \varepsilon\right]}(\lambda) d\left(\mathbf{E}_{\lambda}\right)_{f, f} \\
& \leq \varepsilon B A^{-1} M^{2}\left\langle\mathbf{E}_{\lambda}(\sigma(W)) f, f\right\rangle \\
& =A^{-1} B M^{2} \varepsilon\|f\|^{2}=A^{-1} B M^{2} \varepsilon .
\end{aligned}
$$

Hence, for $\varepsilon \rightarrow 0$,

$$
\inf _{\|f\|_{J} \leq 1}\left(\sum_{i \in I} x_{i}^{2}\left\|Q_{V_{i}} f\right\|_{J}^{2}\right)=0 .
$$

Now, if $\left\{x_{i}, V_{i}\right\}_{i \in I}$ is a frame of subspaces for the singular Krein space $\mathfrak{H}_{W}$ with frame bounds $C, D>0$, then for $f$ given above, by the theorem 2.4 and by (2.6) we arrive to $C=0$, which contradicts that $\left\{x_{i}, V_{i}\right\}_{i \in I}$ is a frame of subspaces for the singular Krein space $\mathfrak{H}_{W}$.

Remark 2.9. A Hilbert space $\mathfrak{H}$ with a $W$-metric arbitrary can be embedded densely in a Krein space $\mathfrak{H}_{W}$. For instance, we note that if $\mathfrak{H}_{W}$ is a regular Krein space, then the frames of subspaces are transferable from $\mathfrak{H}$ to $\mathfrak{H}_{W}$. This happens because $\left(\mathfrak{H}_{W},[\cdot, \cdot]_{J}\right)=\left(\mathfrak{H},[\cdot, \cdot]_{J}\right)$ and the norms $\|\cdot\|,\|\cdot\|_{J}$ are equivalent in $\mathfrak{H}$. But, when the Krein space $\mathfrak{H}_{W}$ is singular, the frames of subspaces are not transferable, because the property $0 \in \sigma(W)$ has strong influence. Hence, we must find a way to extend the frames of subspaces from the Hilbert space $\mathfrak{H}$ to the singular Krein space $\mathfrak{H}_{W}$. 
A way to extend the frames of subspaces from a Hilbert space $\mathfrak{H}$ to a singular Krein space $\mathfrak{H}_{W}$ is made as follows.

Theorem 2.10. Let $\mathfrak{H}_{W}$ be a singular Krein space. There is an invertible operator $\mathcal{U}: \mathfrak{H} \rightarrow \mathfrak{H}_{W}$ such that:

i). If $\left\{x_{i}, V_{i}\right\}_{i \in I}$ is frame of subspaces for the Hilbert space $(\mathfrak{H},\langle\cdot, \cdot\rangle)$, then $\left\{x_{i}, \mathcal{U} V_{i}\right\}_{i \in I}$ is a frame of subspaces for the singular Krein space $\mathfrak{H}_{W}$.

ii). If $\left\{x_{i}, V_{i}\right\}_{i \in I}$ is a frame of subspaces for the singular Krein space $\mathfrak{H}_{W}$, then $\left\{x_{i}, \mathcal{U}^{-1} V_{i}\right\}_{i \in I}$ is a frame of subspaces for the Hilbert space $(\mathfrak{H},\langle\cdot, \cdot\rangle)$.

Proof. In [15] was proved that the operator $\sqrt{|W|}: \mathfrak{H} \subset \mathfrak{H}_{W} \rightarrow \mathfrak{H}$, satisfies $\|\sqrt{|W|} k\|^{2}=\langle\sqrt{|W|} k, \sqrt{|W|} k\rangle=\langle|W| k, k\rangle=\|k\|_{J}^{2}$. i.e., $\sqrt{|W|} \in \mathcal{B}\left(\mathfrak{H}, \mathfrak{H}_{W}\right)$ is an isometry. Therefore, this isometry has an unitary extension on $\mathfrak{H}_{W}$, denoted $\widehat{\sqrt{|W|}}$. Hence, considering $\mathcal{U}=\widehat{\sqrt{|W|}}$, the implications $i$ ) and $i i$ ) are satisfied immediately with help of the theorems 2.4 and 2.6.

The following well known result, see for example [20], is useful for our main purpose.

Proposition 2.11. (Spectral theorem-multiplication operator form) Let $A$ be a bounded self-adjoint operator on a separable Hilbert space $\mathfrak{H}$. Then,

$$
\mathfrak{H}=\bigoplus_{n \in \mathbb{N} \cup\{\infty\}} \mathfrak{H}_{\psi_{n}}
$$

and there are measures $\left\{\mu_{n}\right\}_{n=1}^{N}(N=1,2, \ldots$ or $\infty)$ on $\sigma(A)$ and an unitary operator

$$
T: \mathfrak{H}_{\psi_{n}} \rightarrow L_{2}\left(\sigma(A), d \mu_{n}\right)
$$

such that $\left(T A T^{-1} \psi\right)_{n}(\lambda)=\lambda \psi_{n}, \quad n \in \mathbb{N} \cup\{\infty\}$ where we write an element $\psi \in \bigoplus_{n=1}^{N} L_{2}\left(\sigma(A), d \mu_{n}\right)$ as an $N$-tuple $\left(\psi_{1}(\lambda), \psi_{2}(\lambda), \ldots, \psi_{N}(\lambda)\right)$.

In the previous proposition the realization of $A$ is called a spectral representation, which lead us to the following result.

Theorem 2.12. Let $\mathfrak{H}$ be a separable Hilbert space, let $W$ be the Gram operator defined on $\mathfrak{H}$ such that $0 \in \sigma(W)$. Then the Krein space $\mathfrak{H}_{W}$ has an orthonormal basis of subspaces.

Proof. Note that the Gram operator $W$ is self-adjoint, then by the proposition 2.11 we have $(2.7)$ and there are measures $\left\{\mu_{n}\right\}_{n=1}^{N}(N=1,2, \ldots$ or $\infty)$ on $\sigma(A)$ such that $\mathfrak{H}_{\psi_{n}} \simeq L_{2}\left(\sigma(W), d \mu_{n}\right)$. Hence $\left\{\{1\}, \mathfrak{H}_{\psi_{n}}\right\}_{n \in \mathbb{N} \cup\{\infty\}}$ is a Parseval 
frame of subspaces for the Hilbert space $\mathfrak{H}$ with frame bounds 1 . By the theorem $2.10\left\{\{1\}, \mathcal{U H}_{n}\right\}_{n \in \mathbb{N} \cup\{\infty\}}$ is a Parseval frame of subspaces for the singular Krein space $\mathfrak{H}_{W}$. In fact, $\left\{\{1\}, \mathcal{U H}_{n}\right\}_{n \in \mathbb{N} \cup\{\infty\}}$ is an orthonormal basis of subspaces of $\mathfrak{H}_{W}$. Thus, we conclude that

$$
\mathfrak{H}_{W}=\bigoplus_{n \in \mathbb{N} \cup\{\infty\}} \mathcal{U} \mathfrak{H}_{\psi_{n}}
$$

Remark 2.13. Owing to $0 \notin \sigma(W)$ and by (1.8) we see that

$$
\mathfrak{H}_{W}=\left(\mathfrak{H},[\cdot, \cdot]_{J}\right)=\bigoplus_{n \in \mathbb{N} \cup\{\infty\}}\left(\mathfrak{H}_{\psi_{n}},[\cdot, \cdot]_{J}\right)
$$

Theorem 2.14. Let $\mathfrak{H}_{W}$ be a singular Krein space with Gram operator $W$. Then $\mathfrak{H}_{W}$ has a decomposition as follows

$$
\mathfrak{H}_{W}=\bigoplus_{n \in \mathbb{N} \cup\{\infty\}} \mathfrak{H}_{\psi_{n}}^{W}
$$

Furthermore, there are measures $\left\{\mu_{n}\right\}_{n=1}^{N}(N=1,2, \ldots$ or $\infty)$ on $\sigma(W)$ such that $\mathfrak{H}_{\psi_{n}}^{W} \simeq L_{2}\left(\sigma(W), x d \mu_{n}(x)\right)$ are Krein spaces for every $n \in \mathbb{N} \cup\{\infty\}$.

Proof. (This proof is adapted from [15] ) Since the separable Krein space $\mathfrak{H}_{W}$ is singular, the Gram operator $W$ is such that $0 \in \sigma(W)$. Hence, by the theorem 2.12 the family $\left\{\{1\}, \mathcal{U} \mathfrak{H}_{\psi_{n}}\right\}_{n}$ is an orthonormal basis of subspaces of $\mathfrak{H}_{W}$. Define

$$
\mathfrak{H}_{\psi_{n}}^{W}:=\mathcal{U} \mathfrak{H}_{\psi_{n}}, \quad \forall n \in \mathbb{N} \cup\{\infty\} .
$$

We want to show that $\mathfrak{H}_{\psi_{n}}^{W} \simeq L_{2}\left(\sigma(W), x d \mu_{n}(x)\right)$. Now, fixed $n \in \mathbb{N} \cup\{\infty\}$, $L_{2}\left(\sigma(W), d \mu_{n}\right)$ is a Hilbert space, where $\mu_{n}$ is a Lebesgue measure. Over such Hilbert space we define the bounded and self-adjoint operator given by $\left(W_{x} f\right)(x)=x f(x)$. The linear operator is such that $\operatorname{ker} W_{x}=\{0\}$ due to $\mu_{n}\left(\operatorname{Id}_{\sigma(W)}^{-1}\{0\}\right)=0$, where $\operatorname{Id}_{\sigma(W)} x=x$. Likewise, if $\left(W_{x} f\right)(x)=0$, for all $x \in \sigma(W)$, then given $\varepsilon \in \mathbb{N}$, we take the measurable's sets

$$
M_{\varepsilon}=\{x \in \sigma(W):|f(x)|>\varepsilon\},
$$

and we obtain

$$
\begin{aligned}
0 & =\left\|W_{x} f\right\|^{2}=\int_{\sigma(W) \backslash \operatorname{Id}_{\sigma(W)}^{-1}(\{0\})}|f(x)|^{2}|x|^{2} d \mu_{n} \\
& =\int_{M_{\varepsilon}}|f(x)|^{2}|x|^{2} d \mu_{n}(x) \geq \varepsilon^{2} \int_{M_{\varepsilon} \backslash \operatorname{Id}_{\sigma(W)}^{-1}(\{0\})}|x|^{2} d \mu_{n}(x) .
\end{aligned}
$$


i.e $\mu_{n}\left(M_{\varepsilon}\right)=0, \quad \forall \varepsilon \in \mathbb{N}$. Consequently, if

$$
M^{+}=\{x \in \sigma(W):|f(x)|>0\}=\bigcup_{m \in \mathbb{N}} M_{\frac{1}{m}},
$$

then $\mu_{n}\left(M^{+}\right)=0$. Thus, we conclude $|f|=0$ almost everywhere in $\sigma(W)$. i.e., $f=0$ almost everywhere in $\sigma(W)$.

Now, if over the Hilbert space $L_{2}\left(\sigma(W), d \mu_{n}(x)\right)$ we take the Gram operator $W_{x}$, then

$$
{\overline{L_{2}\left(\sigma(W), d \mu_{n}(x)\right)}}^{\|\cdot\|_{J}} \simeq L_{2}\left(\sigma(W), x d \mu_{n}(x)\right)
$$

is a singular Krein space, where

$$
L_{2}\left(\sigma(W), x d \mu_{n}(x)\right):=\left\{f \in L_{2}\left(\sigma(W), d \mu_{n}\right): \int_{\sigma(W)}|f(x)|^{2}|x| d \mu_{n}(x)<\infty\right\}
$$

and $\|f\|_{J}^{2}=[f, f]_{J}=\left\langle\left|W_{x}\right| f, f\right\rangle=\int_{\sigma(W)}|f(x)|^{2}|x| d \mu_{n}(x)$.

On the other hand, Let $F: L_{2}\left(\sigma(W), d \mu_{n}\right) \longrightarrow L_{2}\left(\sigma(W), x d \mu_{n}(x)\right)$ be a linear operator given by $(F g)(x)=\frac{g(x)}{\psi(x)}$, where the function $\psi(x)$ is measurable and $|\psi(x)|^{2}=|x|$ almost everywhere in $\sigma(W)$. For $n \in \mathbb{N} \cup\{\infty\}$ fixed is satisfies that $\mu_{n}\left(|\psi|^{-1}\{0\}\right)=\mu_{n}\left(\left|\operatorname{Id}_{\sigma(W)}\right|^{-1}\{0\}\right)=0$, consequently

$$
\|F f\|_{J}^{2}=\int_{\sigma(W)}|f(x)|^{2}\left|\psi^{-1}(x)\right|^{2}|x| d \mu_{n}(x)=\int_{\sigma(W)}|f(x)|^{2} d \mu_{n}(x)=\|f\|^{2} .
$$

In addition, the linear operator $F$ has inverse which is well defined and is given by $\left(F^{-1} f\right)(x)=\psi(x) f(x)$.

In conclusion, the theorem is proved from the diagram

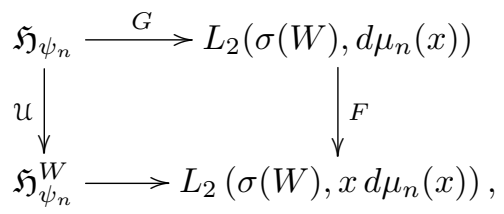

where $G$ is an invertible operator defined in $\mathfrak{H}_{\psi_{n}}$ on $L_{2}\left(\sigma(W), d \mu_{n}(x)\right)$.

\subsubsection{The case: Gram operator $W$ is unbounded.}

Unfortunately, we can not obtain similar results when the Gram operator $W$ on Hilbert space $\mathfrak{H}$ is unbounded. Mainly because the tools used are based 
on specific properties given for the $C^{*}$-algebra $\mathcal{B}(\mathfrak{H})$. In the paper [15] was studied the behavior of the frames in this case. Next, we use such results to show the behavior of the frames of subspaces in the Hilbert spaces with $W$-metric where the Gram operator $W$ is unbounded. For more details see [15].

The Gram operator $W$ is well defined with dense domain $\mathcal{D}_{W} \subsetneq \mathfrak{H}$. Hence, the $W$-metric $[\cdot, \cdot]=\langle W \cdot, \cdot\rangle$ only is defined for $x, y \in \mathcal{D}_{W}=\mathcal{D}_{W^{*}}$. The polar decomposition $(W=J|W|)$ allows us to define

$$
[x, y]_{J}:=\langle|W| x, y\rangle, \quad \forall x, y \in \mathcal{D}_{W},
$$

and by the proposition 1.16 we get

$$
\mathfrak{H}_{W}:=\overline{\mathcal{D}}_{W}\|\cdot\|_{J} .
$$

Proposition 2.15. Let $\left\{x_{i}\right\}_{i \in I} \in \ell_{+}^{\infty}(I)$. Consider a partition $\left\{J_{i}\right\}_{i \in I}$ of $I$ such that $I=\bigsqcup_{i \in I} J_{i}$ and $\left\{k_{i, j}\right\}_{j \in J_{i}}$ a sequence of frame for the Hilbert space $(\mathfrak{H} \cdot\langle\cdot, \cdot\rangle)$ with frame bounds $A_{i}, B_{i}>0$. We assume $V_{i}=\operatorname{span}_{j \in J_{i}}\left\{k_{i, j}\right\}$ and $0<A=\inf _{i \in I} A_{i} \leq B=\sup _{i \in I} B_{i}$ for all $i \in I$. Hence, if $\left\{x_{i}, V_{i}\right\}_{i \in I}$ is a frame of subspaces for the Hilbert space $(\mathfrak{H} .\langle\cdot, \cdot\rangle)$, then $\left\{x_{i}, V_{i}\right\}_{i \in I}$ is not a frame of subspaces for the Krein space $\mathfrak{H}_{W}$.

Proof. We have that $\left\{x_{i} k_{i, j}\right\}_{i \in I, j \in J_{i}}$ is a frame for the Hilbert space $\mathfrak{H}$ (see [9]), but in [15] was proved that $\left\{x_{i} k_{i, j}\right\}_{j \in J_{i}}$ is not a frame for the Krein space $\mathfrak{H}_{W}$ when the Gram operator $W$ is unbounded. Thus, by the proposition 2.5, the family $\left\{x_{i}, V_{i}\right\}_{i \in I}$ is not a frame of subspaces for the Krein space $\mathfrak{H}_{W}$.

Theorem 2.16. Let $\mathfrak{H}_{W}$ be a Krein space where the Gram operator $W$ is unbounded and $0 \notin \sigma(W)$. Let $G: \mathcal{D}_{G} \subset \mathfrak{H}_{W} \rightarrow \mathfrak{H}$ be given by $G=\sqrt{|W|}$, the following statements hold.

i). If $\left\{x_{i}, V_{i}\right\}_{i \in I}$ is frame of subspaces for the Hilbert space $(\mathfrak{H},\langle\cdot, \cdot\rangle)$, then $\left\{x_{i}, G^{-1} V_{i}\right\}_{i \in I}$ is a frame of subspaces for the Krein space $\mathfrak{H}_{W}$.

ii). If $\left\{x_{i}, V_{i}\right\}_{i \in I}$ is a frame of subspaces for the Krein space $\mathfrak{H}_{W}$, then $\left\{x_{i}, G V_{i}\right\}_{i \in I}$ is a frame of subspaces for the Hilbert space $(\mathfrak{H},\langle\cdot, \cdot\rangle)$.

Proof. Since $0 \in \rho(W)$, the linear operator $G$ is invertible ( see [15]). Thus, by the proposition 2.6 , the proof hold.

When the Gram operator $W$ is unbounded with $0 \in \sigma(W)$ we get an analogous result to Theorem 2.10 .

Theorem 2.17. Let $\mathfrak{H}_{W}$ be a Krein space where the Gram operator $W$ is unbounded with $0 \in \sigma(W)$. There is an invertible operator $\mathfrak{U}: \mathfrak{H} \rightarrow \mathfrak{H}_{W}$ such that: 
i). If $\left\{x_{i}, V_{i}\right\}_{i \in I}$ is a frame of subspaces for the Hilbert space $(\mathfrak{H},\langle\cdot, \cdot\rangle)$, then $\left\{x_{i}, \mathcal{U} V_{i}\right\}_{i \in I}$ is a frame of subspaces for the Krein space $\mathfrak{H}_{W}$.

ii). If $\left\{x_{i}, V_{i}\right\}_{i \in I}$ is a frame of subspaces for the Krein space $\mathfrak{H}_{W}$, then $\left\{x_{i}, \mathcal{U}^{-1} V_{i}\right\}_{i \in I}$ is a frame of subspaces for the Hilbert space $(\mathfrak{H},\langle\cdot, \cdot\rangle)$.

Proof. In [15] was proved that for $0 \in \sigma(W)$, the linear operator $G: \mathcal{D}_{G} \subset$ $\mathfrak{H}_{W} \rightarrow \mathfrak{H}$, which is given by $G=\sqrt{|W|}$, has an unique unitary extension $\mathcal{U}:=\widehat{G}: \mathfrak{H}_{W} \rightarrow \mathfrak{H}$. Thence by the proposition 2.6 the statement hold.

\section{Final remarks}

In this paper we study Hilbert spaces with bounded Gram operator. When the Gram operator $W$ is unbounded, our main result is not satisfied whether $\mathfrak{H}$ cannot be decomposed. In such case, we can only state that if $\mathfrak{H}$ satisfies (2.7) in some way, then the decomposition (2.11) is obtained to $\mathfrak{H}_{W}$.

The following open questions arose during the writing of this paper.

i). Is it possible to solve differential equations with frames on some Hilbert or Krein spaces?

ii). In $[1,2,3]$ were studied relations between differential Galois theory and the solution of the Schrödinger equation over separable Hilbert space $\left(L_{2}\right)$, is it possible to obtain similar results in the context of the Krein and Hilbert frames subspaces?.

iii). Is it possible to study partial differential equations with the frames on some Hilbert or Krein spaces?

$i v)$. How can we write a quantum mechanics formalism in the context on the frames of subspaces in Hilbert or Krein spaces?

$v$.) How can we use Banach algebras instead of Hilbert spaces to study the frames theory?

$v i)$. How can we relate the frame theory with Weyl $C^{*}$-algebra?

vii). What happens in the case of tensor product on vector space in the frames theory? 


\section{Acknowledgements}

Thank P. Găvruţa for his observations and recommendations. The first author is partially supported by the MICIIN/FEDER grant number MTM200906973, by the Generalitat de Catalunya grant number 2009SGR859 and by DIDI Universidad del Norte (Raimundo Abello). The second author is supported by CONACYT and CINVESTAV. The third author is supported by Universidad Surcolombiana. The authors thank to Greisy Morillo by their hospitality during the final process of this paper.

\section{References}

[1] P. B. Acosta-HumÁnez, Galoisian approach to supersymmetric quantum mechanics. PhD Thesis, Universitat Politcnica de Catalunya 2009.

[2] P. B. Acosta-Humánez, Galoisian Approach to Supersymmetric Quantum Mechanics: The integrability analysis of the Schrdinger equation by means of differential Galois theory, VDM Verlag Dr Mueller Publishing, 2010.

[3] P. B. Acosta-Humánez, J. J. Morales-Ruiz, J. A. Weil, Galoisian approach to integrability of Schrödinger equation. Reports on Mathematical Physics, 67 (3), (2011), 305-374.

[4] N. Akhiezer, I. Glazman, Theory of linear operators in Hilbert space. Transl. from the Russian. Dover Publications New York, 1993.

[5] T. Ya. Azizov, I. S. Iokhvidov, Linear operators in Hilbert spaces with G-metric. Russ. Math. Surv. 26 (1971), 45-97.

[6] T. YA. AzIzov AND I. S. Iokhvidov, Linear operator in spaces with an indefinite metric. Pure \& Applied Mathematics, A Wiley-Intersciences, Chichester, 1989.

[7] J. Bognar, Indefinite inner product spaces. Springer, Berlin, 1974.

[8] P. G. Casazza, O. Christensen, Weyl Heisenberg Frames for subspaces of $L_{2}(\mathbb{R})$, Proc. Amer. Math. Soc. 129 (2001), 145-154.

[9] P. G. Casazza, G. Kutyniok, Frame of subspaces, arXiv:math/0311384.

[10] O. Christensen, An introduction to frames and Riesz bases, Birkhäuser, Boston, 2003. 
[11] O. Christensen, T. K. Jensen, An introduction to the theory of bases, frames, and wavelets. Technical University of Denmark, Department of Mathemtics, 1999.

[12] I. Daubechies, A. Grossmann, Y. Meyer, Painless nonorthogonal expansions, J. Math. Phys. 27 (1986), 1271-1283.

[13] H. Deguang, K. Kornelson, D. Larson , E. Weber, Frames for undergraduates, Student Mathematical Library, A.M.S Providence, 2007.

[14] R. J. Duffin, A. C. Schaeffer, A class of nonharmonic Fourier series, Trans. Amer. Math. Soc. 72 (1952), 341-366.

[15] K. Esmeral, O. Ferrer, E. Wagner, Frames in Krein spaces arising from a $W$-metric, Banach J. Math., Submited April 2013.

[16] K. Esmeral, Marcos en espacios de Krein, Master thesis, Posgrado Conjunto UNAM-UMSNH, 2011.

[17] P. Găvruţa, On the duality of fusion frames. J. Math. Anal. Appl., 333 (2007), 871-879.

[18] J. I. Giribet, A. Maestripieri, F. Martínez Pería and P. Massey, On a family of frames for Krein spaces, arXiv:1112.1632v1.

[19] E. Christofer, D. Walnut., Continous and discrete wavelet transforms, SIAM. 31 (1989), 628-666.

[20] M. ReED, S. BARRY, Methods of modern mathematical physics, vol. I: Functional Analysis. Academic Press. 1972.

[21] W. Rudin, Functional analysis . McGraw-Hill, New York, 1973.

Acosta-Humánez Primitivo B.,

Department of Mathematics,

Universidad del Atlántico

and Intelectual.Co

Barranquilla, Colombia.

Email: primi@intelectual.co

Esmeral Kevin,

Department of Mathematics,

Centro de Investigación y Estudios Avanzados del Instituto Politécnico

Nacional (CINVESTAV-IPN),

Mexico city, México.

Email: kmesmeral@math.cinvestav.mx 
Ferrer Osmin,

Department of Mathematics,

Universidad Surcolombiana,

Neiva, Colombia.

Email: osmin.ferrer@usco.edu.co 